\title{
Crecimiento postdestete y eficiencia reproductiva de corderas Pelibuey con un sistema de alimentación intensiva ${ }^{\#}$
}

\author{
Postweaning growth and reproductive performance of Pelibuey ewe lambs \\ under an intensive feeding system \\ A Pascual-Córdova ${ }^{a}$, J Oliva-Hernández ${ }^{\text {b, }}$ c $^{*}$ D Hernández-Sánchez ${ }^{\mathrm{d}}$, \\ G Torres-Hernández ${ }^{d}$, ME Suárez-Oporta ${ }^{a}$, JA Hinojosa-Cuéllar ${ }^{b}$

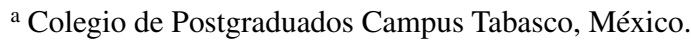 \\ b Universidad Popular de la Chontalpa, México. \\ ${ }^{\mathrm{c}}$ Campo Experimental Huimanguillo, Centro de Investigación Regional Golfo-Centro, INIFAP, México. \\ d Colegio de Postgraduados Campus Montecillo, México.
}

\begin{abstract}
SUMMARY
The aim of this study was to evaluate the influence of age at first mating on the reproductive performance of Pelibuey ewe lambs. The study consisted of two trials: postweaning growth and reproductive handling. Twenty Pelibuey ewe lambs were used which were fed a complete diet throughout the study. In the first trial, the studied variable was the type of birth (TN). Body weight (PV) and daily weight gain (GDP) were studied during 42 days. The TN did not influence $(\mathrm{P}>0.05) \mathrm{PV}$, GDP and age of the ewe lambs at $28 \mathrm{~kg}$ PV. In the second trial, the studied variable was the age of the ewe lamb at $28 \mathrm{~kg}$ PV: YOUNG (139 days) vs MATURE (169 days). PV and ewe lambs in estrus (\%) were studied. In the MATURE group, a 43\% (6/13) of ewe lambs in estrus were detected, whereas in the YOUNG group there were no ewe lambs in estrus (0/7). It was concluded that the type of birth of the ewe lambs had no effect on the postweaning growth performance nor the starting age for reproductive handling at a determined body weight. The starting age for reproductive handling of ewe lambs had an important effect on estrus detection, and there was a high ratio of reproductive failure in young ewe lambs.
\end{abstract}

Palabras clave: cordera, ganancia de peso posdestete, estro, trópico húmedo.

Key words: ewe lamb, postweaning body weight gain, estrus, humid tropic.

\section{INTRODUCCIÓN}

El uso de dietas integrales para alimentar a las corderas en condiciones de estabulación es una opción que ha permitido conocer y aprovechar el potencial de crecimiento de las corderas Pelibuey en sus diferentes etapas de crecimiento (predestete y postdestete). Las consecuencias inmediatas de aplicar este sistema de alimentación han permitido una reducción en los días necesarios para llegar a mercado y la posibilidad de incorporar a las corderas al proceso reproductivo a una menor edad y/o con un mayor peso vivo (Bustamante 2002, Macedo y Arredondo 2008).

Una de las ventajas reproductivas que se han atribuido a las corderas Pelibuey es el alto porcentaje de hembras que muestran el primer estro (89\%) a un peso vivo bajo (21 a $23 \mathrm{~kg}$ ). Sin embargo, la mayor parte de este tipo de información reproductiva se ha generado en condiciones de pastoreo con o sin complementación alimenticia, en

Aceptado: 24.06.2009.

\# FOMIX 2005-1, 16449.

* Km1 carretera Huimanguillo-Cárdenas, Campo Experimental Huimanguillo, INIFAP, Huimanguillo, Tabasco, México; olivajh20@yahoo.com.mx donde es frecuente que las corderas muestren un peso vivo menor a $26 \mathrm{~kg}$ al primer apareamiento y una edad mayor a los diez meses (Castillo 1989, Ramón y Sanginés 2002, Méndez-Sánchez y col 2008). Por ello resulta de interés generar conocimiento que muestre el comportamiento productivo y reproductivo de las corderas Pelibuey al iniciar su primer manejo reproductivo con un peso mayor a los $26 \mathrm{~kg}$ y una edad menor a los ocho meses. La generación de este tipo de conocimiento reproductivo permitiría proponer alternativas de tipo nutricional y/o reproductivo orientadas a incrementar la productividad de las ovejas.

El objetivo general del trabajo consistió en determinar en corderas Pelibuey la influencia de la edad sobre su comportamiento reproductivo cuando estas son alimentadas con una dieta integral desde el destete hasta su primer manejo reproductivo.

\section{MATERIAL Y MÉTODOS}

\section{LOCALIZACIÓN DEL ENSAYO}

Se realizó en la estación experimental Huimanguillo del Instituto Nacional de Investigaciones Forestales, Agrícolas y Pecuarias localizada en Huimanguillo, Tabasco, México (17 $\left.50^{\prime} \mathrm{LN}, 93^{\circ} 23^{\prime} \mathrm{LO}\right)$, a una altitud de 20 metros sobre 
el nivel del mar. El clima de la región es cálido con lluvias todo el año (Af) y la temperatura media anual de $27,8{ }^{\circ} \mathrm{C}$ (“Anónimo" 2007).

\section{PERÍODO EXPERIMENTAL}

El ensayo tuvo una duración de 152 días y fue dividido en una fase preexperimental de 15 días y dos fases experimentales: crecimiento postdestete (95 días) y comportamiento reproductivo (42 días).

\section{ANIMALES}

Se utilizaron 20 corderas Pelibuey de 90 días de edad y un peso vivo de $14,0 \pm 2,0 \mathrm{~kg}$. Las corderas utilizadas se destetaron a los 60 días de edad.

\section{CRECIMIENTO POSTDESTETE}

Se consideró como inicio del estudio el momento en el cual una cordera cumplió 90 días de edad, y como fin, al cumplir los 132 días de edad.

Diseño experimental. Se utilizó un diseño experimental de dos factores con medidas repetidas en un factor (Cody y Smith 1991). El primer factor fue el tipo de nacimiento (simple o doble) del cual provenían las corderas. El segundo factor fue la edad postdestete, que consideró las siguientes edades: 90, 104, 118 y 132 días postdestete. La unidad experimental fue la cordera.

En el tipo de nacimiento simple se incluyeron corderas provenientes de un parto simple y aquellas gemelas que crecieron como corderas únicas hasta el destete. En el tipo de nacimiento doble se consideró a los corderos gemelos, que se mantuvieron vivos desde el nacimiento hasta el destete.

Alimentación. Las corderas se alimentaron de manera individual durante toda la etapa postdestete con una dieta integral (2,8 Mcal EM/kg MS, 19,8\% PC y 93,0\% MS) ofrecida a libre acceso.

Al inicio del estudio se proporcionaron $500 \mathrm{~g} /$ cordera/ día. Posteriormente, la cantidad de alimento se ajustó diariamente, de acuerdo al nivel de consumo experimentado por cada cordera, procurando mantener, al menos, un $10 \%$ de alimento rechazado. La dieta integral se ofreció de 8:00 a 18:00 horas y se cuantificó diariamente el nivel de consumo. Las corderas tuvieron acceso a agua y sal mineral ${ }^{1}$ con monensina sódica. La sal mineral se ofreció en grupo durante el alojamiento nocturno y se cuantificó el consumo diariamente.

Alojamiento. Las corderas se mantuvieron estabuladas en corraletas individuales $\left(3 \mathrm{~m}^{2}\right)$ en un horario de 8:00 a 18:00

\footnotetext{
Foscamag ${ }^{\circledR}$
}

horas. Al finalizar el periodo de alojamiento diurno las corderas se trasladaron a una galera, en donde se alojaron en grupo en un solo corral (3,85 $\mathrm{m}^{2} /$ animal).

Programa sanitario. El control de los parásitos gastrointestinales se efectuó con diferentes fármacos (Febendazole ${ }^{2}$, $1 \mathrm{~mL}$ por cada $10 \mathrm{~kg}$ peso vivo (PV); Levamisol $12 \% \%^{3}, 1 \mathrm{~mL}$ por cada $20 \mathrm{~kg}$ de PV, los cuales se aplicaron a intervalos de 60 días y se alternaron en cada ocasión.

\section{VARIABLES EVALUADAS}

Se evaluaron las siguientes variables: peso vivo, ganancia de peso, ganancia diaria de peso (GDP), consumos de alimento, materia seca, proteína cruda y energía metabolizable.

Peso vivo. Las corderas se pesaron a intervalos de 14 días, entre los 90 y 132 días de edad. Antes de cada sesión de pesaje las corderas fueron sometidas a un período de ayuno de 12 horas. Para obtener el peso vivo de la cordera se utilizó una báscula electrónica ${ }^{4}$, con una precisión de $0,100 \mathrm{~kg}$.

Consumo de alimento. El consumo de la dieta integral por cordera por día se determinó durante tres días consecutivos, procedimiento que se efectuó a intervalos de siete días.

Análisis estadístico. El promedio de consumo de sal mineral se determinó con estadísticos descriptivos. Para establecer la influencia del tipo de nacimiento, edad de la cordera y la interacción entre las variables anteriores sobre las variables productivas se utilizó el procedimiento MIXED (Wang y Goonewardene 2004) con apoyo en el programa computacional SAS versión 8,0 para Windows (SAS Institute, Cary, NC, USA). Para establecer la influencia del tipo de nacimiento sobre las variables cambio de peso total y GDP total se utilizó el procedimiento GLM.

\section{FASE DE COMPORTAMIENTO REPRODUCTIVO}

Animales. Se utilizaron las 20 corderas Pelibuey incluidas en el estudio de crecimiento postdestete. Una vez que estas alcanzaron los 132 días de edad continuaron con el mismo programa de alimentación detallado en la sección de crecimiento postdestete, hasta que éstas alcanzaron los $28 \mathrm{~kg}$ PV, momento de inicio de la etapa de evaluación del comportamiento reproductivo.

Diseño experimental. Se utilizó un diseño de un factor (Cody y Smith 1991). El factor de estudio fue la edad de las corderas, joven y madura, al momento de entrar al manejo reproductivo.

$$
\begin{aligned}
& \text { Panacur } ₫ \\
& \text { Ripercol } ® \\
& \text { Gallagher } ₫
\end{aligned}
$$


Se consideró a una cordera como joven cuando ésta alcanzó $28 \pm 1 \mathrm{~kg}$ PV a una edad menor a los 150 días. Mientras que una cordera madura fue aquella que requirió más de 150 días para alcanzar los $28 \pm 1 \mathrm{~kg} \mathrm{PV}$. Se utilizaron siete corderas jóvenes (139 \pm 2 días de edad) y 13 maduras ( $169 \pm 3$ días de edad).

Manejo reproductivo. Se inició cuando las corderas alcanzaron los $28 \pm 1 \mathrm{~kg} \mathrm{PV}$, independientemente de su edad. Las corderas utilizadas no tuvieron contacto físico y visual con algún carnero adulto desde los 90 días de edad y hasta alcanzar los $28 \mathrm{~kg}$ PV.

La detección de la conducta de estro se realizó dos veces al día (de 7:00 a 7:30 y de 19:00 a 19:30 horas) con apoyo de cuatro carneros (un año de edad) de la raza Blackbelly.

Se consideró a una cordera en estro cuando ésta aceptó el apareamiento con el carnero y mostró alguna de las siguientes conductas de estro: movimiento y agitación frecuente de la cola, levantamiento de las orejas, búsqueda y acercamiento de la hembra al carnero (Jainudeen y Hafez 1996).

Cuando se detectó un hembra en estro se permitió el apareamiento con el mismo carnero durante tres ocasiones a intervalos de 12 horas. Se procuró que los cuatro carneros se aparearan con un número similar de corderas en cada grupo, jóvenes y maduras.

Alimentación. Las corderas recibieron al inicio del manejo reproductivo $1,15 \mathrm{~kg} / \mathrm{animal}$ de la dieta integral; la cantidad de alimento se ajustó diariamente de acuerdo al nivel de consumo mostrado en el día anterior por las corderas, hasta el término del manejo reproductivo. Las corderas dispusieron de agua y sal mineral con monensina sódica ${ }^{5}$ a libre acceso.

\section{VARIABLES EVALUADAS}

La eficiencia reproductiva se evaluó a través de las siguientes variables: edad de las corderas a los $28 \mathrm{~kg}$ PV, condición corporal (cinco escalas, en donde uno corresponde a un animal flaco y cinco al gordo; Russel 1984), GDP durante los primeros 14 días de manejo reproductivo, corderas que mostraron estro y que son apareadas dentro de los 42 días de manejo reproductivo (\%), hembras que repiten estro dentro del período de manejo reproductivo $(\%)$, corderas que no mostraron estro durante el manejo reproductivo (\%), intervalo entre el inicio del manejo reproductivo y el primer estro (días).

Análisis estadístico. Para determinar la influencia de la edad de las corderas sobre la ganancia de peso diaria en los primeros 14 días (20 corderas) de manejo reproductivo se utilizó el procedimiento GLM.

\footnotetext{
Foscamag ${ }^{\circledR}$
}

Las variables que no mostraron una distribución normal se analizaron con la prueba de la suma de rangos de Wilcoxon para datos no pareados (Milton y Tsokos 1987). En el resto de los datos se obtuvieron estadísticos descriptivos. Se consideró el valor de $\mathrm{P}<0,05$ como estadísticamente significativo. Los datos se presentan como media \pm EE.

\section{ESTRUCTURAS OVÁRICAS EN LAS CORDERAS QUE NO PRESENTARON CONDUCTA DE ESTRO}

Animales. Se utilizaron las 14 corderas que no mostraron conducta de estro durante los 42 días de manejo reproductivo: siete de ellas correspondían al grupo de corderas jóvenes y el resto eran maduras. Las medias $\pm \mathrm{EE}$ para edad y PV fueron $194 \pm 4$ días y $34,4 \pm 0,4 \mathrm{~kg}$, respectivamente.

Diseño experimental. Se utilizó un diseño con un factor (Cody y Smith 1991). El factor de estudio fue la edad de las corderas: jóvenes $v s$ maduras.

Las variables estudiadas fueron: peso vivo al finalizar el manejo reproductivo y tipo de estructuras ováricas.

Sacrificio de las corderas. Previo al sacrifico de las corderas, se mantuvieron en ayuno durante 12 horas. Una vez sacrificada la cordera, se procedió a retirar la piel y se realizó una incisión longitudinal a nivel abdominal para obtener el tracto reproductivo completo.

Estructuras ováricas. Los tractos reproductivos completos se conservaron en congelación $\left(0{ }^{\circ} \mathrm{C}\right)$ hasta el momento de su evaluación macroscópica, momento en donde se determinó el tipo de estructuras ováricas presentes en cada ovario. La evaluación se realizó con apoyo de una lupa, un vernier y efectuando una incisión en el diámetro de las estructuras ováricas.

Las estructuras ováricas se clasificaron en dos grupos: lúteas y foliculares con diámetro menor a $5 \mathrm{~mm}$ de diámetro o con diámetro mayor a $5 \mathrm{~mm}$.

Se estableció que una estructura ovárica fue tipo lúteo cuando su apariencia macroscópica correspondió a la de un cuerpo lúteo, con un color entre rosa y rojo, y al efectuar una incisión sobre su diámetro no mostró un antro, ni la presencia de líquido. Mientras que una estructura ovárica de tipo folicular correspondió a aquella estructura con apariencia de folículo, y al efectuar una incisión o punción (con una aguja hipodérmica del número 21) sobre su diámetro se detectó un antro con presencia de líquido folicular.

Análisis estadístico. Para determinar el efecto de la edad de la cordera sobre el peso vivo se utilizó el procedimiento GLM. Las estructuras ováricas presentes en las corderas sacrificadas se describieron con tablas de frecuencia considerando el tratamiento. 


\section{RESULTADOS}

\section{FASE DE CRECIMIENTO POSTDESTETE}

Los promedios en los consumos de materia seca (MS), proteína cruda (PC) y energía metabolizable (EM) durante $\operatorname{los} 42$ días de estudio fueron $547 \pm 20 \mathrm{~g}, 108 \pm 4 \mathrm{~g}$ y $1,547 \pm 0,07 \mathrm{Mcal} /$ cordera/día, respectivamente. El tipo de nacimiento de la cordera $(\mathrm{TN})$ y la interacción $\mathrm{TN} \times$ edad postdestete de las corderas (EDAD) no influyeron (P > 0,05) sobre el consumo de MS, PC y EM. Sin embargo, la EDAD de las corderas afectó $(\mathrm{P}<0,05)$ el nivel de consumo de MS, PC y EM, tal como se muestra en el cuadro 1. Conforme se incrementó la edad de la cordera se aumentó el consumo de MS, PC y EM, detectándose los mayores consumos en los tres nutrimentos estudiados entre los 111 y 132 días de edad con respecto a lo registrado entre los 90 y 96 días de edad.

Las corderas Pelibuey consumieron un promedio de 9,2 $\pm 0,9 \mathrm{~g}$ de sal mineral/cordera/día.

El TN y la interacción TN x EDAD no afectaron $(\mathrm{P}>0,05)$ el peso vivo de las corderas. Sin embargo, las corderas del grupo tipo de nacimiento único mostraron una tendencia $(\mathrm{P}=0,08)$ a lograr un mayor peso vivo al final de los 42 días de estudio con respecto a las corderas gemelas $(24,4 \pm 0,9$ vs 22,0 $\pm 0,7 \mathrm{~kg})$.

La EDAD de las corderas afectó $(\mathrm{P}<0,01)$ su peso vivo, registrándose un incremento significativo en el peso vivo en cada uno de los momentos evaluados en el estudio. Los promedios en los pesos vivos de las corderas a los 90, 104, 118 y 132 días de edad fueron: $15,1 \pm 0,54^{\mathrm{a}}, 17,6 \pm 0,54^{\mathrm{b}}$, $20,2 \pm 0.54^{\mathrm{c}}$ y $23,2 \pm 0,54^{\mathrm{d}}$, respectivamente. Durante los 42 días de estudio las corderas mostraron una ganancia de peso de $8,1 \pm 0,3 \mathrm{~kg}$.

Las corderas mostraron una GDP de $192 \pm 7 \mathrm{~g} /$ cordera. No se detectó influencia $(\mathrm{P}>0,05)$ del TN y de la interacción TN x EDAD en la GDP de las corderas.

La EDAD de las corderas mostró un efecto $(\mathrm{P}<0,05)$ sobre su GDP. Los promedios para la GDP en los períodos 90 a 104 días de edad, 105 a 118 días de edad y 119 a 132 días de edad fueron: $180 \pm 10^{\mathrm{a}}, 187 \pm 10^{\mathrm{a}}$ y $213 \pm 10^{\mathrm{b}}$, respectivamente. En los primeros dos períodos de estudio la GDP fue similar $(\mathrm{P}>0,05)$. Sin embargo, en el tercer período de evaluación la GDP fue mayor en al menos un $14 \%$ a la registrada en los dos primeros períodos.

\section{EFICIENCIA REPRODUCTIVA}

Las corderas iniciaron el manejo reproductivo con un peso vivo, condición corporal y edad de $28,1 \pm 0,1 \mathrm{~kg}$, $3,1 \pm 0,05$ y $159 \pm 4$ días, respectivamente. En el $30 \%$ de las corderas (6/20) se detectó comportamiento de estro durante los 42 días de manejo reproductivo. El peso vivo y edad a la que se detectó su primer estro fue $30,9 \pm 0,8 \mathrm{~kg}$ y $198 \pm 7$ días, respectivamente. En las hembras que mostraron estro el intervalo entre el inicio del manejo reproductivo y el primer estro fue de $24,5 \pm 4,8$ días.
Durante el período de manejo reproductivo las corderas ganaron 5,9 $\pm 0,4 \mathrm{~kg}$ de peso vivo, mostraron una GDP de $140 \mathrm{~g} /$ cordera, concluyeron el manejo reproductivo con $33,9 \pm 0,4 \mathrm{~kg}$ de peso vivo y una condición corporal de $3,5 \pm 0,03$ unidades.

Al iniciar el manejo reproductivo las corderas jóvenes y maduras mostraron un peso vivo similar $(\mathrm{P}>0,05)$ y 30 días de diferencia en la edad ( $\mathrm{P}<0,01)$ (cuadro 2$)$. El tipo de nacimiento de las corderas no ejerció efecto $(\mathrm{P}>0,05)$ sobre la edad a la que alcanzaron los $28 \mathrm{~kg}$ de peso vivo. Las corderas provenientes de un tipo de parto sencillo alcanzaron los $28 \mathrm{~kg}$ a una edad de $153 \pm 6$ días. Mientras que las corderas gemelas a los $162 \pm 5$ días.

Las corderas jóvenes mostraron una mayor GDP en los primeros 14 días del manejo reproductivo $(\mathrm{P}<0,01)$ $\mathrm{y}$ durante todo el manejo reproductivo $(\mathrm{P}<0,05)$ con respecto a las corderas maduras. Sin embargo, en ninguna cordera joven se detectó comportamiento de estro. A diferencia de lo detectado en las corderas jóvenes, el $46 \%$ de las corderas maduras mostraron comportamiento de estro (cuadro 2).

En las corderas maduras el intervalo entre el inicio del manejo reproductivo y el primer estro fue de 25 días y mostró amplia variación $(\mathrm{CV}=48 \%)$.

En el cuadro 3 se muestran las medias para edad, peso vivo y GDP de las corderas al finalizar el manejo reproductivo, considerando su estado reproductivo ( $\sin$ estro o con estro) y su grupo de edad. En las corderas jóvenes no se detectó comportamiento de estro (cuadro 2); sin embargo, en este tipo de ovejas se detectó un mayor $(\mathrm{P}<0,05)$ peso vivo al finalizar el manejo reproductivo y una mayor GDP en los primeros 14 días del manejo reproductivo con respecto al grupo maduro sin estro y maduro con estro.

Las corderas jóvenes, como se indicó previamente, no mostraron comportamiento de estro y fueron las que mayor GDP lograron con respecto a las maduras. En este tipo de corderas el manejo reproductivo se aplicó entre la tercera semana de abril y la cuarta semana de junio de 2007.

En las corderas maduras se detectó mayor variación para iniciar el manejo reproductivo. La primera cordera madura inició el manejo reproductivo en la cuarta semana de abril y las últimas corderas se incorporaron al manejo reproductivo hasta la tercera semana de junio. Las semanas de finalización del manejo reproductivo correspondieron a la primera semana de junio y a la cuarta de julio. Las corderas maduras que mostraron conducta de estro lo presentaron entre la tercera semana de junio y la segunda de julio. Ninguna cordera mostró conducta de estro entre la tercera semana de abril y la segunda de junio de 2007.

Tipo de estructuras ováricas. En las corderas en que no se detectó el estro se detectó que un $50 \%$ de las corderas (7/14) presentó estructuras lúteas y el otro 50\% (7/14) estructuras foliculares. 
Cuadro 1. Consumo de materia seca (MS), proteína cruda (PC) y energía metabolizable (EM) en corderas Pelibuey durante su crecimiento postdestete bajo un sistema de alimentación intensivo (medias de cuadrados mínimos \pm error estándar).

Intake of dry matter, crude protein and metabolizable energy in Pelibuey ewe lambs during their postweaning growth under an intensive feeding system (least squares means \pm standard error).

\begin{tabular}{lcccc}
\hline \multirow{2}{*}{ Edad de las corderas (días) } & \multirow{2}{*}{$\mathrm{N}^{1}$} & \multicolumn{3}{c}{ Consumo de componentes de la dieta integral cordera por día } \\
\cline { 3 - 5 } & & MS, g & PC, g & EM, Mcal \\
\hline 90 a 96 & 20 & $513 \pm 26^{\mathrm{a}}$ & $102 \pm 5^{\mathrm{a}}$ & $1,435 \pm 0,07^{\mathrm{a}}$ \\
97 a 103 & 20 & $532 \pm 26^{\mathrm{a}, \mathrm{b}}$ & $105 \pm 5^{\mathrm{a}, \mathrm{b}}$ & $1,489 \pm 0,07^{\mathrm{a}, \mathrm{b}}$ \\
104 a 110 & 20 & $532 \pm 26^{\mathrm{a}, \mathrm{b}}$ & $105 \pm 5^{\mathrm{a}, \mathrm{b}}$ & $1,488 \pm 0,07^{\mathrm{a}, \mathrm{b}}$ \\
111 a 117 & 20 & $562 \pm 26^{\mathrm{b}, \mathrm{c}}$ & $111 \pm 5^{\mathrm{b}, \mathrm{c}}$ & $1,573 \pm 0,07^{\mathrm{b}, \mathrm{c}}$ \\
118 a 124 & 20 & $590 \pm 26^{\mathrm{c}}$ & $117 \pm 5^{\mathrm{c}}$ & $1,653 \pm 0,07^{\mathrm{c}}$ \\
125 a 132 & 19 & $587 \pm 27^{\mathrm{c}}$ & $116 \pm 5^{\mathrm{c}}$ & $1,643 \pm 0,07^{\mathrm{c}}$ \\
\hline
\end{tabular}

${ }^{1}$ Número de observaciones.

a, b, c Letras diferentes dentro de la misma columna indican diferencias significativas $(\mathrm{P}<0,05)$.

Cuadro 2. Influencia de la edad a la que inician el manejo reproductivo las corderas Pelibuey sobre su eficiencia reproductiva (media \pm error estándar).

Influence of starting age for the reproductive handling of Pelibuey ewe lambs on reproductive performance (mean \pm standard error).

\begin{tabular}{lcc}
\hline & \multicolumn{2}{c}{ Clasificación de las corderas de acuerdo a su edad } \\
\cline { 2 - 3 } Variable & Joven & Madura \\
\hline Edad al iniciar el manejo reproductivo (días) & $139 \pm 2,3^{\text {a }}$ & $169 \pm 3,2^{\mathrm{b}}$ \\
Peso vivo al iniciar el manejo reproductivo (kg) & $28,3 \pm 0,3^{\mathrm{a}}$ & $27,9 \pm 0,1^{\mathrm{a}}$ \\
Ganancia diaria de peso durante los primeros 14 días de manejo reproductivo (g) & $214^{\mathrm{a}}$ (6 $^{\mathrm{a}}$ & $125 \pm 19^{\mathrm{b}}$ \\
Ganancia diaria de peso durante 42 días de manejo reproductivo (g) & $167 \pm 7^{\mathrm{c}}$ & $125 \pm 12^{\mathrm{d}}$ \\
Ovejas detectadas en estro (\%) & $0(0 / 7)^{1}$ & $46(6 / 13)$ \\
Ovejas en las que no se detectó estro (\%) & $100(7 / 7)^{2}$ & $54(7 / 13)$ \\
Número de observaciones & 7 & 13
\end{tabular}

${ }_{\mathrm{a}, \mathrm{b}}$ Letras diferentes dentro de la misma fila indican diferencias significativas $(\mathrm{P}<0,01)$.

${ }^{c, d}$ Letras diferentes dentro de fila indican diferencias significativas $(\mathrm{P}<0,05)$.

${ }^{1}$ entre paréntesis y dentro de la misma fila, la relación número de corderas en estro/número total de corderas en cada grupo de edad.

${ }^{2}$ entre paréntesis y dentro de la misma fila, la relación número de corderas no detectadas en estro/número total de corderas en cada grupo de edad.

Cuadro 3. Peso vivo y ganancia diaria de peso (GDP) de corderas Pelibuey que no mostraron conducta de estro durante un manejo reproductivo de corta duración. Influencia de la edad (media \pm error estándar).

Body weight and daily weight gain of Pelibuey ewe lambs without estrous behavior during a reproductive handling of short duration (mean \pm standard error).

\begin{tabular}{|c|c|c|c|}
\hline \multirow{3}{*}{ Variable } & \multicolumn{3}{|c|}{ Clasificación de las corderas de acuerdo a su estado reproductivo y edad } \\
\hline & \multicolumn{2}{|c|}{ Sin detección de estro } & \multirow{2}{*}{$\frac{\text { Con detección de estro }}{\text { Madura }}$} \\
\hline & Joven & Madura & \\
\hline Edad al finalizar el manejo reproductivo (días) & $181 \pm 2^{\mathrm{a}}$ & $207 \pm 5^{b}$ & $216 \pm 3^{b}$ \\
\hline Peso vivo al finalizar el manejo reproductivo $(\mathrm{kg})$ & $35,3 \pm 0,3^{\mathrm{c}}$ & $33,5 \pm 0,5^{d}$ & $32,8 \pm 0,9^{\mathrm{d}}$ \\
\hline GDP en los primeros 14 días de manejo reproductivo (g) & $214 \pm 16^{\mathrm{c}}$ & $134 \pm 31^{\mathrm{d}}$ & $115 \pm 22^{d}$ \\
\hline GDP durante los 42 días de manejo reproductivo (g) & $167 \pm 7^{\mathrm{c}}$ & $132 \pm 11^{\mathrm{c}}$ & $118 \pm 23^{c}$ \\
\hline Número de observaciones & 7 & 7 & 6 \\
\hline
\end{tabular}

${ }^{\mathrm{a}, \mathrm{b}}$ Letras diferentes dentro de la misma fila indican diferencias significativas $(\mathrm{P}<0,01)$.

${ }^{c, d}$ Letras diferentes dentro de la misma fila indican diferencias significativas $(P<0,05)$. 
Al considerar el grupo de edad, se detectó en las corderas jóvenes, tres casos con estructuras lúteas (una la presentó en ambos ovarios y dos en un solo ovario). En este mismo grupo, una cordera presentó una estructura con aspecto lúteo pero con antro y fluido. En las corderas maduras, se detectaron tres ovejas con estructuras lúteas (dos las presentaron en ambos ovarios).

En el grupo de corderas jóvenes, se detectaron tres hembras con estructuras foliculares con un diámetro menor a los $5 \mathrm{~mm}$ en ambos ovarios. En las corderas maduras, las cuatro hembras mostraron grupos de folículos con un diámetro menor a $5 \mathrm{~mm}$ en ambos ovarios.

\section{DISCUSIÓN}

\section{FASE DE CRECIMIENTO POSTDESTETE}

Aunque existen estudios en donde se evalúa el crecimiento postdestete de las corderas Pelibuey cuando se aplica un manejo intensivo (Bustamante 2002, Macedo y Arredondo 2008), las variables de respuesta estudiadas se limitan, generalmente, a obtener los promedios en el peso vivo y la GDP postdestete lograda en períodos mayores a 90 días. En el presente estudio los consumos de MS, PC y EM resultaron menores a los indicados por Bustamante (2002), quien reportó promedios en los consumos de MS, PC y EM de 770 g, 136 g y 2,7 Mcal, respectivamente. Estas diferencias entre estudios se atribuyen fundamentalmente a la duración del periodo de evaluación del crecimiento postdestete (42 vs 90 días). Adicionalmente, al resultar significativo el efecto EDAD en el presente estudio, los resultados muestran un incremento en el nivel de consumo de MS, PC y EM conforme se aumenta la edad de las corderas.

El consumo de sal mineral en las corderas Pelibuey resultó superior al indicado por Piñeiro-Vázquez y col (2009) en corderas Pelibuey estabuladas y alimentadas con heno de $C$. plectostachyus (2,3 g de sal mineral con monensina/cordera) e inferior al reportado por DíazArcos y col (2008) en corderas Pelibuey en pastoreo con complementación alimenticia (19,2 g de sal mineral con monensina/cordera). Las diferencias en el nivel de consumo de sales minerales entre estudios pueden atribuirse a cambios en el manejo alimenticio (estabulado $v s$ pastoreo) y nivel de respuesta productiva de las corderas. Piñeiro-Vázquez y col (2009) indican una GDP menor a 60 g/cordera, Díaz-Arcos y col (2008) una GDP de 98 $\mathrm{g} /$ cordera, mientras que en el presente estudio se obtuvo una GDP de 192 g/cordera.

Conforme se incrementó la edad de las corderas se registró una mayor GDP; los valores obtenidos son superiores a los indicados por Bustamante (2002) quien obtuvo $132 \mathrm{~g} /$ cordera (2,7 Mcal EM/kg MS y 13,6\% PC) en corderas Pelibuey con un sistema de manejo intensivo, y similares a lo reportado por Macedo y Arredondo (2008) quienes registraron $186 \mathrm{~g} /$ cordera (2,8 Mcal EM/kg MS y $18,8 \%$ PC).

Los valores de GDP postdestete obtenidos en corderas Pelibuey con una dieta integral en este estudio y en el de Macedo y Arredondo (2008) resultan muy superiores a los $98 \mathrm{~g} /$ cordera (300 g/cordera de un complemento alimenticio; 2,6 Mcal EM/kg MS y 24,6\% PC) registrados en corderas Pelibuey en pastoreo con complementación alimenticia (Díaz-Arcos y col 2008) y a los 82 g/cordera obtenidos exclusivamente con el pastoreo en C. plectostachyus (González y col 2002).

\section{EFICIENCIA REPRODUCTIVA}

A pesar de existir estudios reproductivos con corderas Pelibuey desde hace más de 30 años (Castillo y col 1977) no se encontró alguna referencia en donde el manejo reproductivo se inicie con corderas con menos de cinco meses y con $28 \mathrm{~kg}$ de peso. La mayor parte de los estudios reproductivos en corderas Pelibuey se han efectuado con hembras con menos de $26 \mathrm{~kg}$ y más de ocho meses de edad (Castillo 1989, Ramón y Sanginés 2002) o con más de $30 \mathrm{~kg}$ y una edad superior a los ocho meses (Valencia y col 2006, Alvarez y Andrade 2008).

En el presente estudio, todas las corderas mantuvieron una GDP positiva y adecuada condición corporal durante el manejo reproductivo. Sin embargo, un bajo porcentaje de las hembras mostró comportamiento de estro (cuadro 2). A diferencia de lo indicado en este estudio, Sepúlveda y col (2001) indican en corderas Romney Marsh que la GDP postdestete muestra una relación positiva con los porcentajes de actividad ovárica y de estro durante la primera estación reproductiva de las corderas. Mientras que Valencia y col (2006) reportan que las ovejas nulíparas Pelibuey muestran entre diciembre y marzo $\left(19^{\circ} \mathrm{LN}\right)$ una reducción en el porcentaje de actividad estral mensual $(42,8$ a $57,1 \%)$ con relación a lo detectado en los meses de abril a julio (61,9 a 80,9\%). Sin embargo, en el estudio de Valencia y col (2006) se utilizaron ovejas nulíparas que estaban ciclando normalmente al inicio del estudio y contaban con un año de edad y $36 \mathrm{~kg}$. En contraste a lo detectado en el actual estudio, Oliva-Hernández y col (2008) reportan en corderas Pelibuey $\left(27,1 \mathrm{~kg}\right.$ y diez meses de edad; $17^{\circ} 50^{\prime}$ LN, $93^{\circ} 23^{\prime}$ LO) un alto porcentaje de estro (91\%) cuando el manejo reproductivo se realiza en la época de otoño (octubre a noviembre).

Aunque las corderas Pelibuey clasificadas como jóvenes mostraron una mayor GDP durante el manejo reproductivo con respecto a las corderas maduras, ninguna mostró comportamiento de estro. Al parecer, la edad de la cordera al iniciar el manejo reproductivo resultó más determinante que la magnitud en la GDP obtenida. No se dispuso de trabajos en donde se evalúe el comportamiento reproductivo de corderas Pelibuey con menos de cinco meses para poder contrastar los resultados obtenidos en el presente estudio. Sin embargo, Méndez-Sánchez y col 
(2008) muestran que en las corderas Pelibuey x Blackbelly (con ocho meses al iniciar el manejo reproductivo) una diferencia de $4 \mathrm{~kg}$ PV al iniciar el manejo reproductivo $(20,3$ vs $24,3 \mathrm{~kg})$ no resulta determinante en el porcentaje de corderas que muestran conducta de estro $(71,4 v \mathrm{~s} 76,9 \%$, respectivamente) cuando el manejo reproductivo se aplica en los meses de mayo a junio.

En las corderas maduras se detectó un bajo porcentaje de comportamiento de estro, resultado que coincide con lo indicado por Alvarez y Andrade (2008), quienes muestran que en las corderas Pelibuey (ocho a nueve meses de edad) con pesos superiores a $30 \mathrm{~kg}$ y expuestas a un manejo reproductivo de 60 días (abril a mayo; $19^{\circ} 13^{\prime} \mathrm{LN}, 99^{\circ} 8^{\prime}$ LO) se presenta un 50\% de casos de anestro. A diferencia de lo indicado previamente, Ramón y Sanginés (2002) reportan en corderas Pelibuey (un año de edad) un $80 \%$ de hembras en estro al finalizar un manejo reproductivo de 27 días aplicado en el mes de abril $\left(22^{\circ} \mathrm{O}^{\prime} \mathrm{LN} ; 98^{\circ}\right.$ 30' LO).

El intervalo entre el inicio del manejo reproductivo y el primer estro mostró amplia variación, resultados similares han sido indicados previamente (Ramón y Sanginés 2002, Méndez-Sánchez y col 2008). Sin embargo, MéndezSánchez y col (2008) y Oliva-Hernández y col (2008) muestran que cuando la cordera tiene pesos superiores a los $26 \mathrm{~kg}$ se reduce la variación en el intervalo entre el inicio del manejo reproductivo y el primer estro.

La mayor velocidad de crecimiento que mostraron las corderas jóvenes permitió que éstas iniciaran el manejo reproductivo en los meses del año en que existe una mayor duración del fotoperíodo (Oliva y col 2002). Mientras que en el caso de las corderas maduras, en las que se detectó conducta de estro, éste se presentó en los meses de junio y julio. El diseño de este estudio y el tipo de variables estudiadas no permite diferenciar el efecto de edad de la cordera del de duración del fotoperíodo (no evaluado en este estudio). Previamente se indicó (Valencia y col 2006) que en ovejas nulíparas Pelibuey en buena condición corporal y un peso vivo superior a $30 \mathrm{~kg}$ se presenta una reducción en el porcentaje de actividad estral mensual en ciertos meses del año (diciembre a marzo); es probable que este efecto sea de mayor magnitud en corderas con menos de cinco meses, tal como se detectó en este estudio.

Un aspecto no abordado en la cordera Pelibuey es el estudio descriptivo del tipo de estructuras ováricas presentes en las corderas que no muestran comportamiento de estro tras ser expuestas a un manejo reproductivo con apoyo de carnero. En el actual estudio, el 50\% de las corderas que no mostraron comportamiento de estro presentaron estructuras lúteas. Este hallazgo sugiere que el proceso de crecimiento folicular, que culmina en ovulación y desarrollo subsecuente de un cuerpo lúteo, estuvo presente en algunas corderas. Sin embargo, ni el carnero, ni el personal técnico que aplicó el manejo reproductivo fueron capaces de detectar algún comportamiento sexual indicativo de estro. Al respecto, Alvarez y Andrade (2008) muestran diferencias importantes en el intervalo del inicio del manejo reproductivo a la primera ovulación (7,4 días) con el intervalo al primer estro (41,0 días), lo que sugiere que en la cordera Pelibuey se presenta ovulación (al inicio del manejo reproductivo) que no es acompañada de comportamiento de estro, circunstancia que se apoya por los resultados del presente trabajo. En estudios futuros se deberá abordar este tema desde el punto de vista endocrinológico, con el propósito de generar conocimiento que permita explicar la reducción en la proporción de corderas jóvenes que no muestran estro.

En el otro 50\% de las ovejas que no mostraron conducta de estro se detectaron múltiples estructuras foliculares con un diámetro folicular menor a $5 \mathrm{~mm}$, lo que sugiere que los estímulos aplicados a las corderas durante el manejo reproductivo (presencia del carnero, adecuada condición corporal, GDP positiva y un peso vivo que representa al menos un $70 \%$ del peso adulto de la oveja Pelibuey) no resultaron suficientes para estimular el desarrollo folicular que culmine en ovulación y comportamiento de estro.

Se concluye que el tipo de nacimiento de las corderas no influyó sobre su eficiencia de crecimiento postdestete, ni sobre la edad a la que éstas inician el manejo reproductivo a un peso fijo. La edad con la que inician el manejo reproductivo de las corderas tiene un efecto importante sobre el porcentaje de detección del estro, en donde la mayor proporción de fallas reproductivas se presentan en corderas jóvenes.

\section{RESUMEN}

El objetivo del estudio fue evaluar la influencia de la edad al primer apareamiento sobre la eficiencia reproductiva de las corderas Pelibuey. El estudio se dividió en dos ensayos, crecimiento postdestete y manejo reproductivo. Se utilizaron 20 corderas Pelibuey. Las corderas se alimentaron con una dieta integral durante todo el estudio. En el primer ensayo, el factor de estudio fue el tipo de nacimiento (TN). Se evaluó el PV y la ganancia diaria de peso (GDP) durante 42 días. El TN no influyó $(\mathrm{P}>0,05)$ el PV, GDP y edad a la que las corderas alcanzaron $28 \mathrm{~kg}$ PV. En el segundo ensayo, el factor de estudio fue la edad de las corderas al alcanzar los $28 \mathrm{~kg}$ : JOVEN (139 días) vs MADURA (169 días). Las variables evaluadas fueron: PV y corderas en estro (\%). En el grupo MADURA se detectó un 43\% (6/13) de corderas en estro. Mientras que en JOVEN no se detectaron corderas en estro (0/7). Se concluye que el tipo de nacimiento de las corderas no influyó sobre su eficiencia de crecimiento postdestete, ni sobre la edad a la que estas inician el manejo reproductivo a un peso fijo. La edad con la que inician el manejo reproductivo las corderas tiene un efecto importante sobre la detección del estro, en donde la mayor proporción de fallas reproductivas se presentan en corderas jóvenes.

\section{AGRADECIMIENTOS}

El presente material forma parte del proyecto TAB-2005-C06-16449 Fondo Mixto Gobierno del estado de Tabasco y el CONACYT.

\section{REFERENCIAS}

Alvarez L, S Andrade. 2008. El efecto macho reduce la edad al primer estro y ovulación en corderas Pelibuey. Arch Zootec 57, 91-94. 
Anónimo. 2007. Anuario estadístico Tabasco. Instituto Nacional de Estadística, Geografía e Informática. Gobierno del estado de Tabasco, México.

Bustamante JJ. 2002. Crecimiento y finalización de corderos con dietas a base de granos. Campo Experimental "El Verdineño". Instituto Nacional de Investigaciones Forestales, Agrícolas y Pecuarias. Folleto Científico 1, Nayarit, México.

Castillo RH, J Hernández, J Berruecos, J López. 1977. Comportamiento reproductivo del borrego Tabasco mantenido en clima tropical. III Pubertad y duración del estro. Téc Pecu Méx 32, 32.

Castillo RH. 1989. Características reproductivas de la hembra. En: Castellanos AF, Arellano C (eds). Tecnología para la producción de ovejas tropicales. Instituto Nacional de Investigaciones Forestales y Agropecuarias. Organización de las Naciones Unidas para la Agricultura y Alimentación. Mérida, Yucatán, México, y Santiago, Chile, Pp 36-40.

Cody RP, JK Smith. 1991. Analysis of variance. Chapter 7. In: Applied Statistics and the SAS programming language. $3^{\text {rd }}$ ed. North-Holland, Elsevier Science Publishing Co. Inc., USA, Pp 136-159.

Díaz-Arcos F, J Oliva-Hernández, JA Hinojosa-Cuéllar. 2008. Efecto de la suplementación mineral con monensina sódica sobre la eficiencia productiva de corderas Pelibuey. Resúmenes de la XLIV Reunión Nacional de Investigación Pecuaria, Mérida, Yucatán, México, Pp 201.

González RA, BI Martínez, FJA Chávez, HFM Loya, MFA Lucero. 2002. Crecimiento de corderos Pelibuey en Pastoreo. Resúmenes del II Taller Ovino del Golfo y Noreste de México. Universidad Autónoma de Tamaulipas, Tamaulipas, México, Pp 17-20.

Jainudeen MR, ESE Hafez. 1996. Ovejas y cabras. En: Hafez ESE (ed) Reproducción e inseminación artificial en animales. $6^{\mathrm{a}}$ ed. McGrawHill Interamericana, México DF, México, Pp 311-322.

Macedo R, V Arredondo. 2008. Efecto del sexo, tipo de nacimiento y lactancia sobre el crecimiento de ovinos Pelibuey en manejo intensivo. Arch Zootec 57, 219-228.
Méndez-Sánchez JL, J Oliva-Hernández, JA Hinojosa-Cuéllar. 2008. Comportamiento reproductivo de primalas Pelibuey x Blackbelly en un empadre controlado. Resúmenes de la XX Reunión Científica Tecnológica Forestal y Agropecuaria. Universidad Juárez Autónoma de Tabasco, Villahermosa, Tabasco, México, Pp 157-165.

Milton JS, JO Tsokos. 1987. Métodos de distribución libre. Estadística para biología y ciencias de la salud. McGraw-Hill Interamericana, México DF, México, Pp 435-471.

Oliva J, H Mora, JM Sánchez, JA Hinojosa. 2002. Producción de ovinos de pelo en Tabasco. Condiciones climáticas y apareamiento. Kuxulkab', 8, 8-23.

Oliva-Hernández J, JM Zulueta-Rodríguez, JA Hinojosa-Cuéllar. 2008. Evaluación reproductiva de ovejas Pelibuey durante un empadre controlado. Resúmenes del $20^{\circ}$ Encuentro Nacional de Investigación Científica y Tecnológica del Golfo de México. Cd. Madero, Tamaulipas, México, Pp 8.

Piñeiro-Vázquez AT, J Oliva-Hernández, JA Hinojosa-Cuéllar. 2009. Uso de suplementación mineral con monensina sódica en corderas Pelibuey durante el crecimiento postdestete. Arch Med Vet 41, 35-41.

Ramón UJP, GJR Sanginés. 2002. Respuesta al efecto macho de primalas Pelibuey en condiciones de pastoreo y suplementación en trópico. Téc Pecu Méx 40, 309-317.

Russel A. 1984. Body condition scoring of sheep. In Practice, 6, 91-93.

Sepúlveda NG, J Risopatrón, J Oberg, A Neumann. 2001. Suplementación pre y posparto en ovejas. Efecto sobre la pubertad y actividad reproductiva de sus hijas. Arch Med Vet 33, 89-96.

Valencia J, A Porras, O Mejía, JM Berruecos, J Trujillo, L Zarco. 2006. Actividad reproductiva de la oveja Pelibuey durante la época del anestro: Influencia de la presencia del macho. Rev Científ FCVLUZ 16, 136-141.

Wang Z, LA Goonewardene. 2004. The use of MIXED models in the analysis of animal experiments with repeated measures data. Can J Anim Sci 84, 1-11. 